\title{
Entanglement Between Photons that have Never Coexisted
}

\author{
E. Megidish, A. Halevy, T. Shacham, T. Dvir, L. Dovrat, and H. S. Eisenberg \\ Racah Institute of Physics, Hebrew University of Jerusalem, Jerusalem 91904, Israel
}

\begin{abstract}
The role of the timing and order of quantum measurements is not just a fundamental question of quantum mechanics, but also a puzzling one. Any part of a quantum system that has finished evolving, can be measured immediately or saved for later, without affecting the final results, regardless of the continued evolution of the rest of the system. In addition, the non-locality of quantum mechanics, as manifested by entanglement, does not apply only to particles with spatial separation, but also with temporal separation. Here we demonstrate these principles by generating and fully characterizing an entangled pair of photons that never coexisted. Using entanglement swapping between two temporally separated photon pairs we entangle one photon from the first pair with another photon from the second pair. The first photon was detected even before the other was created. The observed quantum correlations manifest the non-locality of quantum mechanics in spacetime.
\end{abstract}

PACS numbers: 03.67.Bg, 42.50.Dv

Entanglement between spatially separated quantum systems is one of the most distinctive results of quantum mechanics. It results in nonclassical correlations between distant systems. Einstein, Podolsky, and Rosen claimed that these instantaneous correlations give rise to a paradox which demonstrates the incompleteness of quantum mechanics [1]. Only after the realization of an experiment suggested by Bell [2, 3], was the nonlocal nature of quantum mechanics widely accepted. Nevertheless, the properties of entanglement still puzzle many researchers.

Single photons are used as quantum particles in many experimental realizations, as they are easily manipulated and preserve their coherence for long times. A common method for generating polarization entangled photon states is using the nonlinear optical process of parametric down-conversion (PDC) in dielectric crystals [4]. In this process, a pump photon splits into two lowerenergy photons while preserving momentum and energy. With this method it is possible to create bright highquality two photon states in any of the four maximally entangled states, also known as the Bell states. For polarized photons these states are

$$
\begin{aligned}
& \left|\phi^{ \pm}\right\rangle=\frac{1}{\sqrt{2}}\left(\left|h_{a} h_{b}\right\rangle \pm\left|v_{a} v_{b}\right\rangle\right), \\
& \left|\psi^{ \pm}\right\rangle=\frac{1}{\sqrt{2}}\left(\left|h_{a} v_{b}\right\rangle \pm\left|v_{a} h_{b}\right\rangle\right),
\end{aligned}
$$

where $h_{a}\left(v_{b}\right)$ represents a horizontally (vertically) polarized photon in spatial mode a (b).

Photons can also be entangled by projection measurements onto maximally entangled states [5]. Bell state measurements with linear optical elements require postselection. They can discriminate simultaneously only between two of the four Bell states [6, 7]. Complete Bell projection can be achieved using nonlinear optics [8], hyper entanglement [9, 10], auxiliary photons [11 13] or path entangled single photons [14]. Bell projections are key ingredients in quantum computation and communication protocols such as teleportation [15] and entanglement swapping [16].

The entanglement swapping protocol entangles two remote photons without any interaction between them. Each of the two photons belongs initially to one of two independent entangled photon pairs (e.g., photons 1 and 4 of the entangled pairs 1-2 and 3-4). The two other photons (2 and 3 ) are projected by a measurement onto a Bell state. As a result, the first two photons (1 and 4) become entangled even though they may be distant from each other. Entanglement swapping is the central principle used in quantum repeaters [17], whose purpose is to overcome the limiting effect of photon loss in long range quantum communication. Previous demonstrations of entanglement swapping [18] and multi-stage entanglement swapping [19], entangled photons that were separated spatially, but not temporally, i.e., all the photons that were entangled, existed and were measured at the same time.

In this work we demonstrate how the time at which quantum measurements are taken and their order, has no effect on the outcome of a quantum mechanical experiment, by entangling two photons that exist at separate times. This is achieved by first creating one photon pair (1-2) and right away measuring photon 1 (see Fig. 1). Photon 2 is delayed until a second pair (3-4) is created and photons 2 and 3 are projected onto the Bell basis. When photon 1 is measured in a certain basis, it does not 'know' that photon 4 is going to be created, and in which basis it will be measured. Nevertheless, photons 1 and 4 exhibit quantum correlations despite the fact that they never coexisted. The possibility of two photons that do not overlap in time, but still exhibit entanglement, was discussed theoretically in a system of atoms and photons [20]. Recently, entanglement swapping was demonstrated with a delayed choice, where all four photons were cre- 


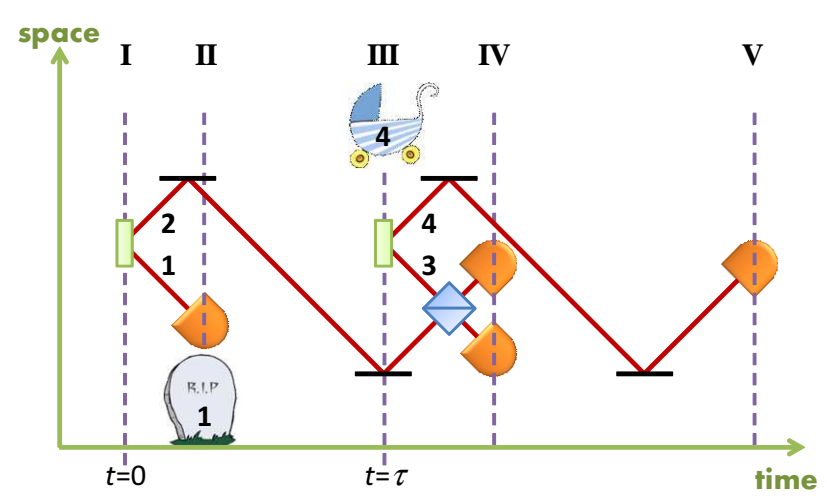

FIG. 1. (color online). Time line diagram. (I) birth of photons 1 and 2. (II) detection of photon 1. (III) birth of photons 3 and 4. (IV) Bell projection of photons 2 and 3. (V) detection of photon 4 .

ated simultaneously, but photons 1 and 4 were measured before a choice had been made whether to entangle them or not 21, 22].

The scenario of time and space separation we create should be compared to the standard two particle entangled state, where the particles are only spatially separated. In the standard entanglement case, the measurement of any one of the particles instantaneously changes the physical description of the other. This result was described by Einstein as "spooky action at a distance". In the scenario we present here, measuring the last photon affects the physical description of the first photon in the past, before it has even been measured. Thus, the "spooky action" is steering the system's past. Another point of view that one can take is that the measurement of the first photon is immediately steering the future physical description of the last photon. In this case, the action is on the future of a part of the system that has not yet been created.

In order to generate consecutive photon pairs at well defined times, a pulsed laser is used to pump a single PDC polarization entangled photon source [4]. It is a probabilistic source, and thus there is a probability that two pairs will be created, each pair from one of two consecutive pulses, separated by the laser period time $\tau$. The four-photon state is

$$
\begin{gathered}
\left|\psi^{-}\right\rangle_{a, b}^{0,0} \otimes\left|\psi^{-}\right\rangle_{a, b}^{\tau, \tau}= \\
\frac{1}{2}\left(\left|h_{a}^{0} v_{b}^{0}\right\rangle-\left|v_{a}^{0} h_{b}^{0}\right\rangle\right) \otimes\left(\left|h_{a}^{\tau} v_{b}^{\tau}\right\rangle-\left|v_{a}^{\tau} h_{b}^{\tau}\right\rangle\right),
\end{gathered}
$$

where the subscripts are the spatial mode labels and the superscripts are the time labels of the photons. In order to project the second photon of the first pair and the first photon of the second pair onto a Bell state, the former is delayed by $\tau$ in a delay line. The same delay is also applied to the second photon of the second pair and the

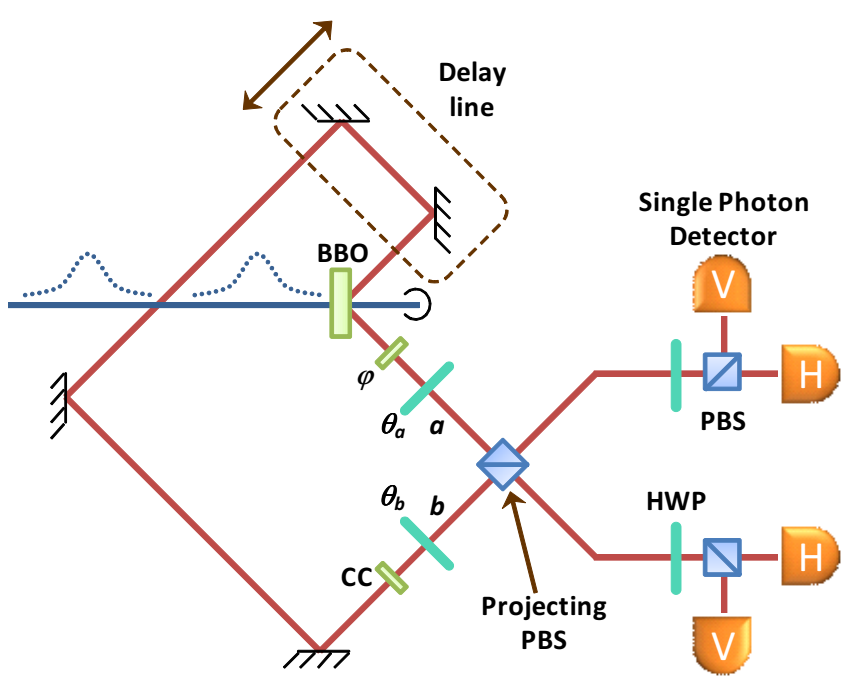

FIG. 2. (color online). The experimental setup (see text for details).

resulting state can be reordered and written as

$$
\begin{aligned}
\left|\psi^{-}\right\rangle_{a, b}^{0, \tau} \otimes\left|\psi^{-}\right\rangle_{a, b}^{\tau, 2 \tau}= & \frac{1}{2}\left(\left|\psi^{+}\right\rangle_{a, b}^{0,2 \tau}\left|\psi^{+}\right\rangle_{a, b}^{\tau, \tau}\right. \\
& -\left|\psi^{-}\right\rangle_{a, b}^{0,2 \tau}\left|\psi^{-}\right\rangle_{a, b}^{\tau, \tau} \\
& -\left|\phi^{+}\right\rangle_{a, b}^{0,2 \tau}\left|\phi^{+}\right\rangle_{a, b}^{\tau, \tau} \\
& \left.+\left|\phi^{-}\right\rangle_{a, b}^{0,2 \tau}\left|\phi^{-}\right\rangle_{a, b}^{\tau, \tau}\right) .
\end{aligned}
$$

When the two photons of time $\tau$ (photons 2 and 3 ) are projected onto any Bell state, the first and last photons (1 and 4) collapse also into the same state and entanglement is swapped. The first and last photons, that did not share between them any correlations, become entangled.

According to this description, the timing of each photon is merely an additional label to discriminate between the different photons, and the time in which each photon is measured has no effect on the final outcome. The first photon from the first pair (photon 1) is measured even before the second pair is created (see Fig. 11). After the creation of the second pair, the Bell projection occurs and only after another delay period is the last photon from the second pair (photon 4) detected. Entanglement swapping creates correlations between the first and last photons non-locally not only in space, but also in time. Quantum correlations are only observed a posteriori, after the measurement of all photons is completed.

We realized this scenario with the experimental setup presented in Fig. 22 23]. Polarization entangled photon pairs are created by non-collinear type-II PDC [4]. A pulsed Ti:Sapphire laser source with a $76 \mathrm{MHz}$ repetition rate is frequency doubled to a wavelength of $390 \mathrm{~nm}$ and an average power of $400 \mathrm{~mW}$. The laser beam is corrected for astigmatism and focused on a $2 \mathrm{~mm}$ thick $\beta-\mathrm{BaB}_{2} \mathrm{O}_{4}$ (BBO) crystal. Half wave plates (HWP) and compensating crystals (CC) correct for temporal walk-offs. Tilting 
of the compensating crystal in path $a$ is used to control the phase $\varphi$ of the state, e.g., for $\varphi=\pi$ the resulting state is $\left|\psi^{-}\right\rangle$[4]. The $780 \mathrm{~nm}$ wavelength down-converted photons are spatially filtered by coupling them into and out of single-mode fibers, and spectrally filtered by using $3 \mathrm{~nm}$ wide bandpass filters (not shown).

One photon from the first pair is delayed until another pump pulse arrives at the generating crystal by a $31.6 \mathrm{~m}$ (105 ns) free-space delay line. The delay is built from high reflecting dielectric mirrors, with an overall transmittance higher than $90 \%$ after 10 reflections. Less than $10 \%$ of the signal is sampled into a single mode fiber as a feedback signal that is used to stabilize the delayed beam's spatial properties, by tilting a piezo-mounted mirror in the middle of the delay line. We chose the delay length to be the time between eight consecutive laser pulses, in order not to lose signal due to the dead-time of the singlephoton detectors (Perkin Elmer SPCM-AQ4C), and to provide enough time for the measurement of the first photon before the second pair is created. The delayed photon of the first pair and the non-delayed photon of the second pair are projected onto a Bell state by combining them at the projecting polarizing beam-splitter (PBS) (see Fig. 2) [5]. We post-select the cases where each photon exits this PBS at a different port. We ensure that the photons are indistinguishable, i.e., no information is available as to whether both were transmitted or both were reflected. After passing through the PBS, the photons are rotated by HWPs to the $|p / m\rangle=\frac{1}{\sqrt{2}}(|h\rangle \pm|v\rangle)$ polarization basis. For reasons of complementarity, we also define the circular polarization basis $|r\rangle=\frac{1}{\sqrt{2}}(|h\rangle+i|v\rangle)$ and $|l\rangle=\frac{1}{\sqrt{2}}(i|h\rangle+|v\rangle)$. When the polarizations of the middle photons are correlated ( $h h$ or $v v$ ) they are projected onto a $\left|\phi^{+}\right\rangle_{a, b}^{\tau, \tau}$ state. When they are anti-correlated ( $h v$ or $v h$ ) they are projected onto a $\left|\phi^{-}\right\rangle_{a, b}^{\tau, \tau}$ state.

In order to fully characterize the first and last photons' state, a quantum state tomography (QST) procedure is required 24]. Generally, such a procedure involves independent polarization rotations of each of the photons involved. The photons that we are interested in characterizing (1 and 4) are measured by the projecting PBS. Before this PBS, they share the same paths with the other two photons that entangle them (2 and 3 ). Thus, arbitrary rotations of HWP angles $\theta_{a}$ and $\theta_{b}$ will also affect the required entangling projection and the whole projected state. It would appear that fast polarization rotators are required to selectively rotate some photons and not others before the projecting PBS. We have found a way to circumvent this by relying on the entangled nonlocal nature of the initial photon pairs.

The polarization of a single photon that belongs to an entangled pair is undefined (completely mixed) until the polarization of the other photon is measured. Furthermore, the polarization of each of the photons depends on the specific basis in which the polarization of the other
TABLE I. The values of the three angles and their corresponding projection measurements that were used for a complete QST of the first and last photons. The first 5 settings generate projections onto orthogonal states. The other four project onto elliptical polarizations, where $\left|e_{x y}\right\rangle=\alpha(|x\rangle+|y\rangle)$ and $\alpha$ is the normalization factor.

\begin{tabular}{ccccl}
\hline \hline & $\theta_{a}$ & $\varphi$ & $\theta_{b}$ & Polarization states \\
\hline 1 & 0 & 0 & 0 & $|h h\rangle,|h v\rangle,|v h\rangle,|v v\rangle$ \\
2 & $22.5^{\circ}$ & 0 & 0 & $|p p\rangle,|m m\rangle,|m p\rangle$ \\
3 & $22.5^{\circ}$ & $90^{\circ}$ & 0 & $|p l\rangle,|m l\rangle$ \\
4 & $22.5^{\circ}$ & $90^{\circ}$ & $-22.5^{\circ}$ & $|l l\rangle,|l r\rangle$ \\
5 & 0 & $90^{\circ}$ & $22.5^{\circ}$ & $|r m\rangle$ \\
6 & 0 & $90^{\circ}$ & $11.25^{\circ}$ & $\left|e_{h r} e_{h m}\right\rangle$ \\
7 & $11.25^{\circ}$ & $90^{\circ}$ & 0 & $\left|e_{h m} e_{h l}\right\rangle$ \\
8 & $11.25^{\circ}$ & $90^{\circ}$ & $45^{\circ}$ & $\left|e_{v p} e_{v l}\right\rangle$ \\
9 & $45^{\circ}$ & $90^{\circ}$ & $11.25^{\circ}$ & $\left|e_{v r} e_{v p}\right\rangle$ \\
\hline \hline
\end{tabular}

photon is measured. As the projection of the two middle photons is always on the $h / v$ basis, their rotation is manifested as a nonlocal rotation of the first and last photons. Thus, the polarization of the first photon is affected both locally by the phase $\varphi$ and the HWP angle $\theta_{a}$ and non-locally by the HWP angle $\theta_{b}$. Similarly, the polarization of the last photon is affected locally by the HWP angle $\theta_{b}$ and non-locally by the phase $\varphi$ and the HWP angle $\theta_{a}$. The overall operations on these photons are

$$
\begin{aligned}
& \hat{M}_{1}\left(\theta_{a}, \varphi, \theta_{b}\right)=\hat{R}\left(\theta_{a}\right)\left[\begin{array}{cc}
1 & 0 \\
0 & e^{i \varphi}
\end{array}\right] \sigma_{x} \hat{R}\left(\theta_{b}\right) \sigma_{x}, \\
& \hat{M}_{4}\left(\theta_{a}, \varphi, \theta_{b}\right)=\hat{R}\left(\theta_{b}\right) \sigma_{x}\left[\begin{array}{cc}
1 & 0 \\
0 & e^{i \varphi}
\end{array}\right] \hat{R}\left(\theta_{a}\right) \sigma_{x} .
\end{aligned}
$$

where $\hat{R}(\theta)$ is a HWP rotation, and the $\sigma_{x}$ operation is a pauli rotation due to the anti-correlation in the initial $|\psi\rangle$ state. Even though the two rotations are not independent, it is possible to rotate the first and last photons differently. Nevertheless, not any arbitrary rotation is possible due to the rotations dependence. We have found 9 angle settings that enable the projection onto 16 independent states and a complete QST of the first and last photons (see Table ).

The density matrix of the first and last photons was constructed, conditioned on the outcome of the projection of the two photons of time $\tau$. If the projected photons were measured in the $\left|\phi^{+}\right\rangle_{a, b}^{\tau, \tau}$ state, the first and last photons were entangled in the $\left|\phi^{+}\right\rangle_{a, b}^{0,2 \tau}$ state (see Fig. 3a). Alternatively, if the projected photons were measured in the $\left|\phi^{-}\right\rangle_{a, b}^{\tau, \tau}$ state, the first and last photons were entangled in the $\left|\phi^{-}\right\rangle_{a, b}^{0,2 \tau}$ state (see Fig. 3b). The fidelity between the measured and the theoretical density matrices is $(77 \pm 1) \%$. Errors were calculated using 


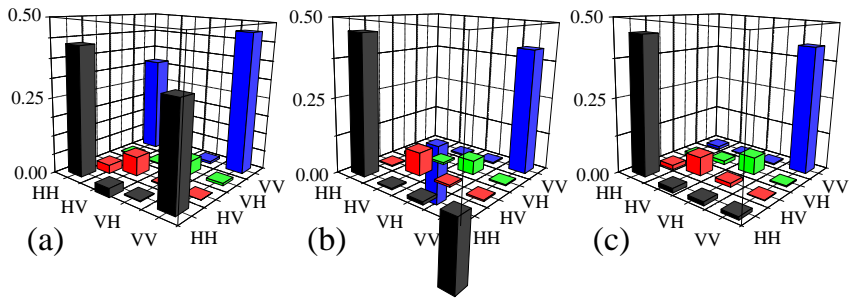

FIG. 3. (color online). Real parts of the density matrices of the first and last photons: (a) when the two middle photons are projected onto the $\left|\phi^{+}\right\rangle$state, (b) when the two middle photons are projected onto the $\left|\phi^{-}\right\rangle$state, and (c) when the projection fails due to temporal distinguishability.

a bootstrapping test, assuming a Poissonian error distribution. The total fourfold count rate was $12 \mathrm{~Hz}$ and each polarization setting was integrated over 6 minutes.

One can also choose to introduce distinguishability between the two projected photons. In this case, the phase between the two terms of the $|\phi\rangle$ projected state is undefined, resulting in a mixture of $\left|\phi^{+}\right\rangle$and $\left|\phi^{-}\right\rangle$in the projected state, and the first and last photons do not become quantum entangled but classically correlated. We observed this when we introduced a sufficient temporal delay between the two projected photons (see Fig. 3r). It is also evidence that the first and last photons did not somehow share any entanglement before the projection of the middle photons.

The fidelity of the measured entanglement is not perfect due to several causes. It is affected in two ways by the entanglement quality of the original photon pairs. The PDC process produces some spectral distinguishability between the photons that reduces the quality of pair entanglement [25], which in turn, limits the maximal quality of the swapped entanglement. In addition, it reduces the quality of the nonlocal rotations that are used in the QST procedure. Another cause for reduced swapping fidelity is the presence of higher order events. We estimate this effect to reduce the fidelity by $\sim 2 \%$.

The scenario we have created is very likely to occur in future quantum repeater realizations [17]. When only one entangled photon reaches a node, it is delayed or stored in a quantum memory until a second photon from another entangled pair arrives. During this waiting period, the distant photon from the first pair can already be used. Only after the arrival of a photon from the second pair, are the two photons projected onto a Bell state and entanglement is generated a posteriori between the other two distant photons.

In conclusion, we have demonstrated quantum entanglement between two photons that do not share coexistence. Although one photon is measured even before the other is created, full quantum correlations were observed by measuring the density matrix of the two photons, con- ditioned on the result of the projecting measurement. This is a manifestation of the non-locality of quantum mechanics not only in space, but also in time. The inductive nature of the setup that was used suggests that it is possible in principle to use it to observe multiple stage entanglement swapping.

The authors thank the Israeli Science Foundation for supporting this work under Grant 546/10.

[1] A. Einstein, B. Podolsky, and N. Rosen, Phys. Rev. 47, 777 (1935).

[2] J. S. Bell, Physics 1, 195 (1964).

[3] A. Aspect, P. Grangier, and G. Roger, Phys. Rev. Lett. 47, 460 (1981).

[4] P. G. Kwiat, K. Mattle, H. Weinfurter, A. Zeilinger, A. V. Sergienko, and Y. H. Shih, Phys. Rev. Lett. 75, 4337 (1995).

[5] H. Weinfurter, Europhys. Lett. 25, 559 (1994).

[6] L. Vaidman and N. Yoran, Phys. Rev. A59, 116 (1999).

[7] N. Lütkenhaus, J. Calsamiglia, and K.-A. Suominen, Phys. Rev. A59, 3295 (1999).

[8] Y.-H. Kim, S. P. Kulik, and Y. Shih, Phys. Rev. Lett. 86, 1370 (2001) .

[9] J. T. Barreiro, T. C. Wei, and P. G. Kwiat, Nature Physics 4, 282 (2008).

[10] C. Schuck, G. Huber, C. Kurtsiefer, and H. Weinfurter, Phys. Rev. Lett. 96, 190501 (2006)

[11] Z. Zhao, A.-N. Zhang, Y.-A. Chen, H. Zhang, J.-F. Du, T. Yang, and J.-W. Pan, Phys. Rev. Lett. 94, 030501 (2005).

[12] P. Walther and A. Zeilinger, Phys. Rev. A72, 010302(R) (2005).

[13] W. P. Grice, Phys. Rev. A 84, 042331 (2011).

[14] D. Boschi, S. Branca, F. De Martini, L. Hardy, and S. Popescu, Phys. Rev. Lett. 80, 1121 (1998).

[15] C. H. Bennett, G. Brassard, C. Crépeau, R. Jozsa, A. Peres, and W. K. Wootters, Phys. Rev. Lett. 70, 1895 (1993).

[16] M. Żukowski, A. Zeilinger, M. A. Horne, and A. K. Ekert, Phys. Rev. Lett. 71, 4287 (1993).

[17] H.-J. Briegel, W. Dür, J. I. Cirac, and P. Zoller, Phys. Rev. Lett. 81, 5932 (1998).

[18] J.-W. Pan, D. Bouwmeester, H. Weinfurter, and A. Zeilinger, Phys. Rev. Lett. 80, 3891 (1998).

[19] A. M. Goebel, C. Wagenknecht, Q. Zhang, Y.-A. Chen, K. Chen, J. Schmiedmayer, and J.-W. Pan, Phys. Rev. Lett. 101, 080403 (2008).

[20] R. Wiegner, C. Thiel, J. von Zanthier, and G. S. Agarwal, Opt. Lett. 36, 1512 (2011).

[21] A. Peres, J. Mod. Opt 47, 139-143 (2000).

[22] X.-S. Ma, S. Zotter, J. Kofler, R. Ursin, T. Jennewein, Ĉ. Brukner, and A. Zeilinger, Nature Physics 8, 480 (2012).

[23] E. Megidish, T. Shacham, A. Halevy, L. Dovrat, and H. S. Eisenberg, Phys. Rev. Lett. 109, 080504 (2012).

[24] D. F. V. James, P. G. Kwiat, W. J. Munro, and A. G. White, Phys. Rev. A 64, 052312 (2001).

[25] P. J. Mosley, J. S. Lundeen, B. J. Smith, P. Wasylczyk, A. B. U'Ren, C. Silberhorn, and I. A. Walmsley, Phys. Rev. Lett. 100, 133601 (2008). 